\title{
NOUVELLE
}

\section{Les cellules dendritiques \\ folliculaires des ganglions stockent du VIH-1 infectieux malgré les traitements antirétroviraux}

Hélène Dutartre
Équipe oncogenèse rétrovirale, équipe labellisée «Ligue Nationale Contre le Cancer », centre international de recherche en infectiologie Inserm U1111 - CNRS UMR5308, École normale supérieure de Lyon, université Lyon 1, LabEx ECOFECT - Eco-evolutionary dynamics of infectious diseases, 46, allée d'Italie, 69007 Lyon, France. helene.dutartre@ens-lyon.fr
> Malgré le succès des traitements antirétroviraux (ART), qui permettent de réduire la quantité du virus de l'immunodéficience humaine de type 1 (VIH-l) dans le sang à des niveaux indétectables, l'infection par ce virus n'est jamais complètement éliminée. La levée du traitement induit en effet systématiquement un rebond viral, suggérant qu'il existe des compartiments inaccessibles à la chimiothérapie, ou que le virus persiste à l'état latent dans les cellules, ces deux hypothèses n'étant pas mutuellement exclusives [1] $(\rightarrow)$.

$\rightarrow$ Voir la Synthèse de T. Cherrier et al., $\mathrm{m} / \mathrm{s} \mathrm{n} \mathrm{n}^{\circ} 3$ mars 2010, page 291

Des biopsies effectuées sur des ganglions de patients infectés par VIH-l et traités par ART, montrent que des lymphocytes $T$ $\mathrm{CD}^{+}$(cellules cibles du virus) sont toujours infectés, et que la réplication virale reste forte dans ces compartiments qui semblent protégés de la chimiothérapie [2].

Les cellules à l'origine de cette forte réplication virale dans les ganglions sont connues depuis longtemps. Il s'agit des lymphocytes Thelpers folliculaires (Tfh), une sous-population de lymphocytes $T$ $\mathrm{CD}^{+}$. De façon surprenante, contrairement aux autres lymphocytes $\mathrm{T} \mathrm{CD}^{+}$, le nombre de Th augmente au cours de l'infection mais cela uniquement dans les ganglions [3]. C'est le virus lui-même qui modifie les propriétés des Tfh infectés et induit leur prolifération [4]. Ces cellules pourraient également être le siège de la latence virale: elles expriment un facteur de transcription, BCL6 (B-cell lymphoma 6), responsable du maintien de leur phénotype, qui est capable de se fixer également sur le promoteur viral (LTR, long terminal repeat) et de réprimer la transcription virale [5]. Mais pourquoi ces cellules ne seraientelles pas éliminées par la chimiothérapie ou le système immunitaire une fois infectées? L'explication tient certainement à leur localisation dans les ganglions, et plus particulièrement dans la zone $\mathrm{B}^{1}$ (Figure IA; pour revue voir [6]). En effet, cette zone est inaccessible aux cellules T CD8 cytotoxiques, qui ne possèdent pas les récepteurs de chimiokines nécessaires leur permettant d'y migrer. Les CD8 cytotoxiques ne peuvent donc pas lyser les Tfh infectés [7]. La latence virale pourrait être une autre raison qui permettrait d'expliquer la persistance du virus dans ces cellules malgré la chimiothérapie. Cependant, dès leur sortie des ganglions, les cellules infectées sont éliminées par les cellules $\mathrm{T} \mathrm{CD}^{+}$cytotoxiques, comme cela est démontré dans le modèle de macaques infectés par le virus de l'immunodéficience simienne (SIV), l'analogue du VIH chez le singe [8]. Une troisième raison pourrait être la persistance de nouvelles infections des cellules Tfh rendue possible par la présence de virus infectieux qui seraient stockés dans les ganglions

\footnotetext{
${ }^{1}$ Les ganglions sont composés de zones, B (ou cortex), dans leur partie externe, contenant des cellules B organisées en follicules primaires et secondaires, et $\mathrm{T}$ (ou paracortex) dans
} leur partie profonde et contenant des cellules $\mathrm{T}$. au sein des cellules dendritiques folliculaires (FDC) (pour revue voir [8]). Ces cellules stromales ${ }^{2}$ sont capables de retenir des complexes immuns antigéniques dans lesquels les antigènes $(\mathrm{Ag})$ sont recouverts de molécules du complément. Les FDC capturent ces complexes via l'interaction entre le récepteur CD21 (ou CR2, récepteur au complément) et la molécule du complément C3d qui recouvre les $\mathrm{Ag}$. Ces complexes immuns sont apportés dans les ganglions soit par la circulation lymphatique, soit par les lymphocytes B naïfs, capables, eux aussi, de fixer des antigènes opsonisés ${ }^{3}$ après interaction avec CD21 (Figure 1). Les particules virales du VIH-l sont des cibles des molécules du complément. Cependant leur opsonisation ne réduit pas leur infectivité, mais au contraire pourrait l'augmenter. On sait depuis presque 30 ans que les FDC sont capables de retenir le VIH-1 dans les ganglions, sans toutefois connaître les mécanismes qui en sont responsables. Les FDC peuvent concentrer I'ARN du virus jusqu'à 40 fois plus que les cellules lymphocytaires, et cela pendant des années, malgré la chimiothérapie antivirale. Ces cellules ont ainsi été suspectées d'être le réservoir viral permettant d'expliquer le rebond de la virémie après l'arrêt des traitements ART. Pourtant les FDC ne sont pas productivement infectées,

\footnotetext{
${ }^{2}$ Issues de la moelle osseuse.

${ }^{3}$ L'opsonisation correspond au recouvrement de la membrane d'une cellule ou bactérie par des molécules (opsinines) afin de favoriser la phagocytose.
} 


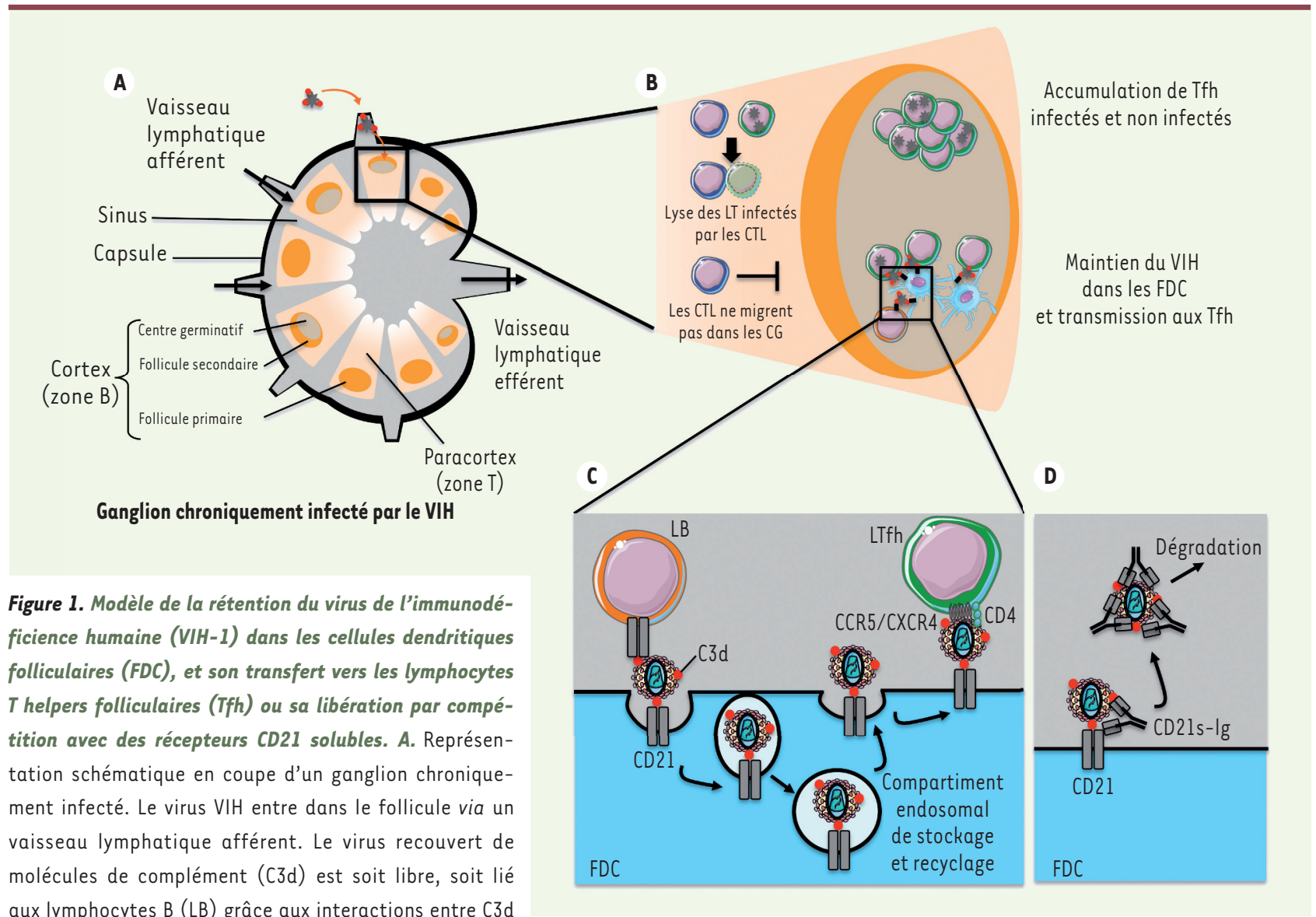
aux lymphocytes $B(L B)$ grâce aux interactions entre C3d et CD21 (récepteur au complément de type 2, non représenté pour plus de clarté). B. Agrandissement d'un centre germinatif (CG) et de la zone extra-folliculaire. Les lymphocytes $C D 8^{+}$cytotoxiques $(C T L)$ restent confinés dans la zone extra folliculaire, où ils peuvent lyser spécifiquement les cellules infectées. Dans le centre germinatif, les interactions entre les FDC et les cellules Tfh permettent le transfert du VIH-1 et la prolifération/ accumulation des Tfh. C. Agrandissement de l'interaction entre les FDC et les cellules B d'une part et les Tfh d'autre part, lors du transfert du VIH-1. Le virus lié au CD21 via C3d est internalisé par les FDC dans des compartiments endosomaux de recyclage, puis présenté au Tfh lors de son passage à la membrane. $D$. Modèle de la compétition avec le CD2l soluble (CD21s-lg) qui permet de purger les compartiments endosomaux du virus (adapté de [6] et [9]). LT : lymphocyte T; CCR5/CXCR4 : C-C chemokine receptor 5/C-X-C chemokine receptor 4.

c'est-à-dire qu'elles ne produisent pas de nouvelles particules virales. Comment le virus peut-il donc être maintenu dans ces cellules et surtout comment peut-il être transmis aux cellules Tfh des ganglions, malgré une thérapie antivirale? L'étude de Heesters et collaborateurs [9] apporte des éléments de réponse. Cette équipe avait montré de façon très élégante, chez la souris, que les FDC étaient capables de recycler les complexes immuns dans un modèle non viral [10]. Après la capture d'Ag modèles recouverts de $\mathrm{C} 3 \mathrm{~d}$ et «présentés » à la surface des lymphocytes B par le CD21, ces Ag sont stockés par les FDC, dans des compartiments neutres où l'on retrouve aussi le récepteur de la transférine mais pas LAMP-1 (lysosomal-associated membrane protein 1 ), le marqueur des endosomes tardifs/lysosomes. En utilisant la même méthodologie, les auteurs ont d'abord confirmé que les FDC humaines, purifiées à partir de ganglions prélevés chez des individus non infectés par le VIH-1, capturent et recyclent des complexes immuns présentés par des lymphocytes B allogéniques. Ensuite, et de façon plus intéressante, les auteurs ont utilisé des FDC purifiées à partir de ganglions inguinaux (situés au niveau de l'aine) humains de patients infectés par VIH-l et traités par ART pendant plusieurs années, dans lesquels ils ont recherché la présence du virus. Comme les ganglions sont composés de plusieurs types cellulaires, dont des lymphoctytes T potentiellement infectés (Figure lB), la pureté des populations de FDC a été contrôlée. La protéine virale de capside (la p24) a été détectée, par immunofluorescence, dans $23 \%$ des FDC issues de ces patients qui étaient pourtant sous traitement ART depuis 8 ans en moyenne. Dans ces FDC marquées (et donc comportant la protéine p24), entre une et trois vésicules contenaient des protéines virales, dans des compartiments de 
recyclage tel que défini par leur marquage avec la transférine et l'absence de marquage par LAMP-1. Ainsi, comme les FDC murines, les FDC humaines accumulent des complexes immuns dans des compartiments de recyclage. La présence de I'ARN viral a également été recherchée par RT-qPCR (reverse transcription quantitative polymerase chain reaction) et a permis de confirmer la présence du virus dans les FDC. En revanche, aucun ADN viral n'a été détecté dans ces cellules, ce qui confirme qu'elles ne sont pas infectées par VIH-1, mais suppose qu'elles sont capables de stocker le virus pendant des années. La présence de I'ARN et des protéines virales dans les FDC de patients ne signifie pourtant pas que celui-ci est infectieux, question particulièrement importante puisque les FDC proviennent de patients sous ART pour lesquels la charge virale est indétectable dans le sang. Aussi, dans une troisième série d'expériences, Heesters et ses collaborateurs ont recherché si le virus retenu dans les FDC, était bien infectieux. Pour cela, les FDC isolées des ganglions de patients infectés ont été cocultivées avec des lymphocytes $T$ $\mathrm{CD}^{+}$hétérologues préalablement activés, procédé qui permet d'augmenter la sensibilité des cellules à l'infection par le VIH-1. Curieusement, les virus stockés dans les FDC restent infectieux, malgré le traitement ART, comme l'atteste la présence aussi bien de l'ARN viral que de I'ADN viral intégré dans les lymphocytes T cibles. Cette série d'expériences confirme ainsi la fonction de « réservoir » des FDC. Puisque le VIH-l est maintenu dans des compartiments qui recyclent, c'est-àdire dont le contenu retourne en surface, les auteurs ont recherché s'il était possible de purger ces compartiments en capturant les virus lors de leur passage en surface. Des études antérieures avaient en effet montré qu'un traitement avec des anticorps dirigés contre le CD2l permettait de réduire la quantité de virus détecté dans les ganglions [11]. Les FDC de patients infectés par VIH-l ont donc été traités avec une forme soluble du CD21 (le CD2ls). Dans ces conditions, la quantité de virus présents dans les vésicules des FDC issues de ganglions des patients, telle que détectée par imagerie ou par RT-qPCR, est largement réduite. En revanche, le traitement des FDC des patients avec des anticorps neutralisants, dirigés contre $\mathrm{VIH}$, reste inefficace. Ceci suggère que seul le récepteur $\operatorname{CD} 21$ soluble est capable de purger le virus des vésicules, probablement par compétition lors du transit à la membrane plasmique, au cours de son recyclage. De la même manière, le CD2ls permet d'éliminer le virus fixé à la surface des lymphocytes B. Enfin, et comme attendu, les FDC traitées avec le CD2ls ne transmettent plus de virus aux lymphocytes cibles, ce qui confirme l'efficacité de la purge des compartiments réservoirs des FDC. Le traitement combiné d'un récepteur CD2l soluble et d'une chimiothérapie ART permettrait donc d'éliminer le virus stocké dans les cellules réservoirs des ganglions, de limiter la transmission du virus aux cellules cibles (les Tfh des ganglions) et donc de diminuer les rechutes à l'arrêt des traitements.

Ce travail permet donc de mieux comprendre le stockage du virus $\mathrm{VIH}-1$ infectieux dans les ganglions en élucidant le mécanisme de son maintien dans les FDC (Figure 1C). Ce travail illustre aussi un nouvel exemple de détournement de la fonction «normale » des cellules par le VIH-1. En capturant et stockant des virus circulants avant les traitements ART, les FDC contribuent certes à la création d'anticorps neutralisants de haute affinité [12], mais également au transfert du virus aux Tfh des centres germinatifs, cibles privilégiées du virus. Mais en élucidant le mécanisme de capture et de maintien dans les vésicules des FDC, dépendant du récepteur CD21, les travaux d'Heester et ses collègues permettent de proposer des alternatives thérapeutiques afin d'éliminer totalement le virus de l'organisme, et pourquoi pas, envisager une guérison totale, après un traitement combinant la chimiothérapie et une prise de CD21 soluble. Les modalités de production de cette molécule, sa stabilité et sa disponibilité dans les ganglions restent encore à déterminer, mais cette alternative est un grand espoir pour les patients. $\diamond$

HIV- 1 is stored by follicular dendritic cells in lymph nodes even under antiviral treatments

\section{LIENS D'INTÉRÊT}

L'auteur déclare n'avoir aucun lien d'intérêt concernant les données publiées dans cet article.

\section{RÉFÉRENCES}

1. Cherrier T, Le Douce V, Redel L, et al. Un virus tapi dans l'ombre : les bases moléculaires de la latence du VIH-1. Partie II : Ia réactivation de la latence du $\mathrm{VIH}-1$ et ses implications thérapeutiques. Med Sci (Paris) $2010 ; 26: 291-6$

2. Folkvord JM, Armon C, Connick $\varepsilon$. Lymphoid follicles are sites of heightened human immunodeficiency virus type l (HIV-1) replication and reduced antiretroviral effector mechanisms. AIDS Res Hum Retrovir $2005 ; 21: 363-70$.

3. Lindqvist M, van Lunzen J, Soghoian DZ, et al. Expansion of HIV-specific T follicular helper cells in chronic HIV infection. J Clin Invest 2012 ; 122 : 327180

4. Kohler SL, Pham MN, Folkvord JM, et al. Germinal center $\mathrm{T}$ follicular helper cells are highly permissive to HIV-l and alter their phenotype during virus replication. J Immunol 2016 ; 196 : 2711-22.

5. Kaczmarek K, Morales A, Henderson AJ. T cell transcription factors and their impact on HIV expression. Virology $2013 ; 2013: 41-7$.

6. Miles B, Connick $\varepsilon$. TFH in HIV latency and as sources of replication-competent virus. Trends Microbiol 2016 ; 24 : 338-44.

7. Fukazawa $Y$, Lum R, Okoye AA, et al. B cell follicle sanctuary permits persistent productive simian immunodeficiency virus infection in elite controllers. Nat Med $2015 ; 21$ : 132-9.

8. Heesters BA, Myers RC, Carroll MC. Follicular dendritic cells: dynamic antigen libraries. Nat Rev Immunol 2014 ; 14 : 495-504.

9. Heesters BA, Lindqvist M, Vagefi PA, et al. Follicula dendritic cells retain infectious HIV in cycling endosomes. PLoS Pathog 2015; 11 : e1005285.

10. Heesters BA, Chatterjee P, Kim YA, et al. Endocytosis and recycling of immune complexes by follicular dendritic cells enhances $B$ cell antigen binding and activation. Immunity 2013; 38:1164-75.

11. Kacani L, Prodinger WM, Sprinzl GM, et al. Detachment of human immunodeficiency virus type 1 from germinal centers by blocking complement receptor type 2.J Virol $2000 ; 74: 7997-8002$.

12. Klein F, Halper-Stromberg A, Horwitz JA, et al. HIV therapy by a combination of broadly neutralizing antibodies in humanized mice. Nature 2012 ; 492 : 118-22. 